Figure 2 shows the distribution of all total alley speeds under the three reinforcement conditions in .01-sec class intervals for all four $\mathrm{Ss}$ in the DNC-PRF-CRF condition and for the yoked PRF-PRF-CRF condition. The cutoff speed below which reinforcement was presented ( $1 \mathrm{ft} / \mathrm{sec}$ ) is shown by the vertical line in the top panel. Clearly, in the DNC-PRF-CRF condition, the distribution of DNC speeds falls to the left of the PRF and CRF distributions, and an insignificant percentage of speeds in any reinforcement condition overlaps DNC in the two groups. The distributions for PRF-PRF-CRF are virtually identical. These data provide further support from within-S experiments for Logan's (1960) contention that low speed in DNC training is not simply a function of low frequencies of reinforcement, but reflects adjustment to the negatively correlated conditions of reinforcement.

A breakdown of the total alley speed plotted in Fig. 1 into speeds in the five separate runway segments in each alley showed that the low total alley speeds late in acquisition in the DNC component were due almost entirely to very low speeds in the start segment. For all Ss, these low first-segment speeds contrasted with relatively high, and virtually identical, speeds in the start measures in the PRF and CRF components. The four other runway measures were highly similar in the three reinforcement conditions. In two Ss, distinct "rituals" developed in the DNC component in the start segment: S 1 bit at a small opening in the floor between the start segment and the runway, and S 2 ran back into the start chamber after inspecting the runway stimulus. These behaviors were not observed in the start segment of the CRF or PRF alleys. Idiosyncratic behaviors of this sort were observed regularly in our earlier experiments (Amsel and Rashotte, 1969; Rashotte \& Amsel, 1968).

In our earlier experiments, we found that responses learned under DNC conditions in DNC-CRF acquisition emerged in the extinction of responding in the CRF alley (e.g., Rashotte \& Amsel, 1968), and we argued that the transfer of DNC acquisition performance to CRF extinction might be due, at least in part, to the same factors that are responsible for PRF-like extinction in the CRF alley after within-S CRF-PRF acquisition (e.g., Amsel, Rashotte, \& MacKinnon, 1966). That is, just as the slow responding of $\mathrm{DNC}$ acquisition emerges in the CRF alley during extinction, so the persistent fast responding learned in the $\mathrm{PRF}$ alley

emerges to maintain a high level of responding in the $C R F$ alley during extinction.

Now that we know that Ss can learn a slow response in a DNC alley and fast ones in PRF and CRF alleys under DNC-PRF-CRF conditions, we would of course like to know, in line with the above reasoning, whether either or both of the intermittently reinforced responses transfer to the CRF alley in extinction. Or, what are the conditions under which DNC or PRF responding is dominant over the other? The present experiment has shown that it is possible to train rats simultaneously under these reinforcement conditions in the runway, but the pilot-study nature of the extinction phase in the present experiment was not adequate to answer the above questions under experimental conditions similar to those in our earlier experiments. The PRF and CRF responses have nearly identical response topographies so that it is difficult to determine if the persistent PRF response transfers to the CRF extinction without a control group run in all three alleys under CRF. To answer questions about extinction, we will need much larger numbers of $S s$ and the CRF control condition.
AMSEL, A MACKINNON, J. R. RASHOTTE, M. E., \& SURRIDGE, C.T. Partial reinforcement (acquisition) effects within subjects. Journal of the Experimental Analysis of Behavior, 1964, 7. 135-138.

\title{
Response patterns as a factor in choice
}

\author{
PETER KILLEEN* \\ Arizona State University, Tempe, Ariz. 85281
}

In the first experiment, pigeons were given a choice between two fixed-interval 40-sec schedules. Varying the pause length by introducing stimulus changes at various times in the intervals had no effect on choice behavior. In the second experiment, pigeons were given a choice between two delays of reinforcement, and preference for the shorter delay was found to be more extreme than predicted by the matching relation. Taken together, the experiments show that preference for a schedule is more dependent on the immediacy of reinforcement than on the presence or nature of responding during the schedule chosen, and that in fixed delays of reinforcement, just as in fixed intervals, preference is more extreme than predicted from matching.

Recent studies of preference for schedules of reinforcement have shown that the major determinant of an animal's choice is how long it will have to wait to get the programmed reinforcement. The response rate engendered by the schedule chosen has scant effect on preference (Killeen, 1968). 1 The presence of stimuli

* This research was conducted with the support of NSF Grants GB 3121 and GB 3723 while I was an NIMH Predoctoral Fellow at Harvard University. Reprints are available from the Department of Psychology, Arizona State University, Tempe, Arizona 85281 . correlated with the schedule in effect is also of little importance. Neuringer (1969) pitted delays of reinforcement against equal fixed-interval (FI) schedules and found about $55 \%$ preference for the FI schedule over a large range. When a stimulus other than blackout was used to signal the delay, he found indifference between equal-valued fixed intervals and fixed delays.

For many schedules the control exerted by delay of reinforcement (or, more conveniently, immediacy of reinforcement, which is the reciprocal of the delay) is beautifully simple; 
preference for a schedule equals the relative immediacy of reinforcement provided by it. This relation has been shown to hold when animals choose between variable-interval (VI), variable-ratio, and fixed-interval vs variable-interval schedules. Chung \& Herrnstein (1967) have shown it to hold for fixed delays of reinforcement. Unfortunately, the relation fails in one of the simplest of all cases: When given a choice between two FI schedules, pigeons prefer the shorter FI more than predicted from the simple matching rule (Killeen, 1970).

Why do pigeons match for delays of reinforcement but not for FIs? We have seen that it is probably not the response requirement, perse, which causes the extremity of preference for short FI schedules, yet the scalloped or break-and-run character of responding typically found on FI schedules may produce cues which affect preference. Pigeons typically pause for about half of the fixed interval before they start to respond, and then their rate is similar to that found on VI schedules of length comparable to the remaining half of the interval (Schneider, 1969). We may thus analyze responding on each key in the typical choice experiment as a three-component chain-initial choice link, self-imposed timeout (post-"reinforcement" pause) upon entry to the terminal link, and terminal link response run. The middle link in this chain may cause the choice behavior which brings it about to deviate from matching because, as a signal for timeout, it differentially weakens behavior on the longer schedule. Conversely, these pauses may act as discriminative stimuli associated with chain schedules of reinforcement and have a conditioned reinforcing strength conferred on them as some function of the delay between their offset and the occurrence of food. In either case, whether the net effect is deleterious or beneficial, the role of the pause may be ascertained by manipulating exteroceptive stimuli while keeping the schedules of reinforcement constant.

\section{EXPERIMENT 1 \\ Method}

Six adult male White Carneaux pigeons, all with previous experience in choice experiments, were maintained at $80 \%$ of their free-feeding weights.

The experimental procedure conformed to the standard concurrent chain design, which consists of an initial choice link and a terminal reinforcement link. In the choice link, responses of at least $15 \mathrm{~g}$ force to either of two Gerbrands pigeon keys changed the color of that key from blue to white (left key) or from blue to green (right key), with the other key going dark. Those changes were scheduled by concurrent VI 1-min schedules. In both terminal links, the first response after $40 \mathrm{sec}$ provided $3.5-\mathrm{sec}$ access to grain. The only difference between the two terminal links was the response-contingent change of key color about half-way through the interval. For the left key, the first response $20 \mathrm{sec}$ after entry into the terminal link changed the color of the key from white to red. For the right key, the first response $x$ sec after entry into the terminal link changed the color of the key from green to orange. The distribution of stimulus change intervals $(x)$ for the right key was approximately normal, with a mean of $20 \mathrm{sec}$, and ranged from 2 to $38 \mathrm{sec}$.

The pigeons were run for 12 sessions, in each of which they received 48 reinforcements. After this, they were run for another 10 sessions, with the variable stimulus change on the left key and 20-sec stimulus change on the right key.

\section{Results and Discussion}

The response pattern in the terminal links came under the control of the key color change. Average pause on the variable key was 13.0 and $13.1 \mathrm{sec}$, while the pause on the fixed key was 17.7 and 17.2 sec. These figures are the means over the last three sessions of the time to the second response in the terminal link. In both the initial experiment and the replication, the animals at first showed a preference for the fixed key, but became indifferent between the schedules within a week. Mean preferences for the variable key were .526 and .506 , with ranges of .10 and .17 . There was no systematic correlation between relative pause and preference.

The indifference shown by pigeons to these two schedules cannot be attributed to inattention to the key color change, since pause length changed appropriately. Choice behavior seems to be unaffected by the response pattern in the schedules chosen. How, then, do we account for the difference in the nature of preference between FIs and delays of reinforcement? At this point, it will be useful to attempt a replication of Chung and Herrnstein's results, and then to systematically modify the terminal links to find at what point preference becomes exaggerated.

\section{EXPERIMENT 2 Method}

Four White Carneaux pigeons, all with previous experience in choice experiments, though not in the above experiment, were maintained at at $80 \%$ of their free-feeding weights.

The procedure was similar to that of Chung \& Herrnstein (1967). Responses on either of two white response keys in the initial link would occasionally cause all lights in the chamber to go off for 5 sec (left key) or 20 sec (right key), after which the grain hopper and light would be activated for 3 sec. Entry into the terminal link (the 5-or 20 -sec delays) was programmed by independent concurrently running VI 1 -min schedules. Sessions terminated after 60 reinforcements.

After 17 days, the delays for the two keys were reversed, with the 20 -sec delay occurring in the left terminal link and the 5-sec delay in the right terminal link. This condition was run for 32 days. There was a 1-sec changeover delay in effect for the first 10 days of each condition.

\section{Results and Discussion}

The preferences for the longer delay, as measured by the relative number of responses emitted on that key in the initial link, were .236, .094, .084 , and .055 in the first condition and $.085, .102, .137$, and .043 for the same pigeons in the replication. These data are means from the last 5 days at each condition. In all cases but one, the preferences are more extreme than predicted by matching to the relative immediacy of reinforcement. The average value of .105 is closer to that found for equivalent FI schedules (.07, Killeen, 1970) than to the matching value of .20 , and represents a failure to replicate Chung and Herrnstein's data.

It is not clear what differences in procedure might have caused the differences in results. The data, however, are consistent with those studies that have indicated that there is little difference between the effects of fixed-delay schedules and fixed-interval schedules on the behavior which precedes them.

\section{REFERENCES}

CHUNG, S. H., \& HERRNSTEIN, R. J. Choice and delay of reinforcement Journal of the Experimental Analysis of Behavior, 1967, 10,67-74.

KILLEEN, $\mathrm{P}$. Response rate as a factor in choice. Psychonomic Science, 1968, 12, 34.

KILLEEN, P. Reinforcement frequency and contingency as factors in fixed-ratio behavior. Journal of the Experimental Analysis of Behavior, $1969,12,391-395$

KILLEEN, P. Preference for fixed-interval schedules of reinforcement. Journal of the Experimental Analysis of Behavior. $1970,13,127-131$.

NEURINGER, A. Delayed reinforcement versus reinforcement after a fixed interval. Journal of the Experimental Analysis of Behavior, $1969,12,375-383$.

SCHNEIDER, B. A. A two-state analysis of fixed-interval responding in the pigeon. Journal of the Experimental Analysis of Behavior, 1969, 12, 677-687.

\section{NOTE}

1. Schedules which command less than maximal rates may do so by reinforcing behaviors other than recorded responses, so that response rate does not reflect the total amount of effort emitted during a schedule (Killeen, 1969). 